\title{
Dual-specific T cells combine proliferation and antitumor activity
}

\author{
Michael H. Kershaw, Jennifer A. Westwood, and Patrick Hwu \\ Published online 4 November 2002; doi:10.1038/nbt756
}

\begin{abstract}
An effective immune response against cancer requires the activation and expansion of specific T cells. Tumor antigens, however, are generally poor immunogens. To achieve expansion of tumor-reactive T cells in vivo, we used a strategy of generating dual-specific T cells that could respond to a powerful immunogen while also possessing tumor reactivity. We generated dual-specific $T$ cells by genetic modification of alloreactive $T$ cells with a chimeric receptor recognizing folate-binding protein, an ovarian cancer-associated antigen. Mouse dualspecific $T$ cells responded in vitro to both allogeneic antigen and tumor cells expressing folate-binding protein, and expanded in number in vivo in response to immunization with allogeneic cells. Most importantly, the combination of dual-specific T cells and immunization had an antitumor effect in vivo. We also generated human dual-specific T cells and characterized the dual-specific nature of individual clones. Assigning the tasks of expansion and tumor reactivity to different receptors within the same lymphocyte may help to overcome the problem of poor immunogenicity of tumor antigens.
\end{abstract}

Directing the immune system against cancer has long been a goal of immunologists. The many tumor-associated antigens (TAA) with little or no expression in normal tissues are attractive targets that might provide a highly specific antitumor response with little toxicity to normal tissue $^{1}$. In humans, a wide variety of tumor antigens with defined T-cell reactivity are known. Some are currently being assessed as vaccines in clinical trials ${ }^{2,3}$, but responses to these vaccines have been infrequent ${ }^{4}$. One reason for this limited success may be the absence of sufficient numbers of tumor-reactive T cells. Indeed, tumor antigens are thought to be poor immunogens.

We and others have investigated the potential of adoptive immunotherapy using large numbers of in vitro-activated T cells in an attempt to enhance the number and activity of $T$ cells potentially applicable as therapeutics for a variety of viral and malignant disease $\mathrm{e}^{5-8}$. In mouse tumor models, adoptive immunotherapy can effectively mediate antitumor effects ${ }^{9,10}$. Humans have also benefited from treatment that includes adoptive transfer of autologous tumor-reactive $\mathrm{T}$ cells $\mathrm{s}^{7,8}$, although responses are restricted to only a proportion of patients suffering from one of a limited range of malignancies. This limited success may result from poor persistence of tumor-specific $T$ cells, because large numbers of these activated cultured cells do not survive long in vivo after adoptive transfer ${ }^{11}$.

With these considerations in mind, we developed a strategy involving the generation of dual-specific $\mathrm{T}$ cells that are able to proliferate in vivo in response to a powerful immunogen and that also recognize tumor antigen. In this approach, the endogenous T-cell receptor (TCR) provides activation and proliferative signals to $\mathrm{T}$ cells in response to the immunogen, whereas tumor specificity is provided by genetic modification with a chimeric receptor that recognizes TAA. The immunogen used in this study was allogeneic major histocompatibility complex (MHC) present on the surface of allogeneic cells. We chose to use allogeneic antigen (alloantigen) because it is well documented and provides a powerful stimulus that induces rapid activation and expansion of alloreactive $\mathrm{T}$ cells, which are found in large numbers and have diverse functions and phenotypes in most individuals ${ }^{12,13}$. The chimeric tumor-reactive receptor used was made up of an extracellular domain composed of single-chain antibody specific for the human ovarian TAA folate-binding protein (FBP), and an intracellular signaling domain derived from the $\gamma$ chain of the human Fc receptor complex, which we have previously shown to induce cytokine secretion and lytic capability in T cells ${ }^{14}$.

In this study, we examined the ability of adoptively transferred dual-specific $\mathrm{T}$ cells to expand in vivo in response to allogeneic immunization and to inhibit tumor growth following immunization with allogeneic cells.

\section{Results}

Phenotype and reactivity of alloreactive T cells. In our initial experiments, we characterized the $\mathrm{T}$ cells that were generated in a mixed lymphocyte reaction (MLR). Flow cytometric analysis of $\mathrm{T}$ cells derived from Thy $1.1^{+}$C57BL/6 mice indicated that more than $95 \%$ were $\mathrm{CD}^{+}$in all experiments. In addition, they had high levels of Thy- $1.1^{+}$expression, allowing efficient tracking of alloreactive T cells after adoptive transfer into Thy- $1.2^{+}$congenic recipients in subsequent experiments. We analyzed the function of these $\mathrm{T}$ cells using a cytokine secretion assay (Fig. 1). T cells raised in the MLR secreted large amounts of interferon- $\gamma$ (IFN- $\gamma$ ) in response to the allogeneic targets $\mathrm{CT} 26$ and $\mathrm{BALB} / \mathrm{c} 3 \mathrm{~T} 3$ but not the syngeneic targets B16, 24JK, or C57BL/6 splenocytes, thereby demonstrating specific alloreactivity of the T cells. Allogeneic stimulation was necessary for the generation of alloreactive $\mathrm{T}$ cells, as $\mathrm{T}$ cells raised against a melanocyte differentiation antigen, Trp-2, secreted IFN- $\gamma$ only in response to peptide-pulsed splenocytes and not in response to allogeneic targets.

Expansion of alloreactive $\mathrm{T}$ cells in vivo. We next determined whether cultured alloreactive $\mathrm{T}$ cells could expand in vivo in response to immunization with allogeneic cells. We injected Thy-1.1 ${ }^{+}$ 


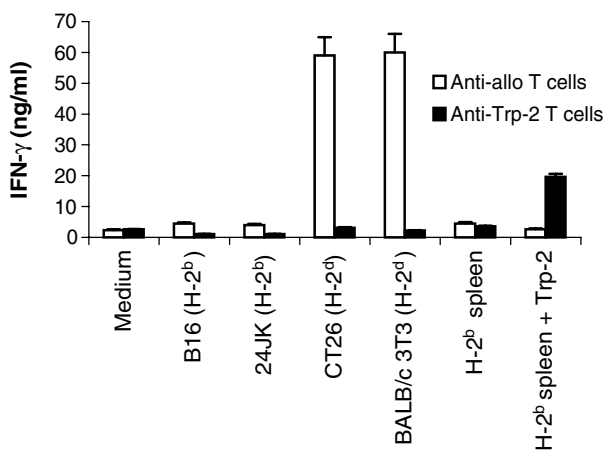

Target cell

Figure 1. Reactivity of anti-allogeneic mouse T cells. Anti-allogeneic T cells respond to the allogeneic cell lines CT26 and 3T3, but not to the syngeneic lines B16 and 24JK or to syngeneic splenocytes, as demonstrated by IFN- $\gamma$ release after overnight coculture. The requirement for allogeneic stimulation to generate alloreactive T cells is supported by the absence of alloreactivity of $T$ cells raised against the melanocyte differentiation antigen Trp-2. Results from one representative experiment of three are shown.

alloreactive $\mathrm{T}$ cells intravenously into Thy- $1.2^{+}$congenic recipient mice, immunized some groups of mice with allogeneic splenocytes, and harvested spleen, lung, and blood 11 days after T-cell transfer. Whereas only small numbers of Thy- $1.1^{+}$cells were detected in the absence of immunization (Fig. 2A), a much higher percentage of adoptively transferred $\mathrm{T}$ cells was detected following subcutaneous immunization, demonstrating that in vitro-cultured, adoptively transferred lymphocytes responded to immunization. The magnitude of expansion in response to immunization was consistently greater than tenfold, with adoptively transferred cells constituting up to $5 \%$ of total cells in tissues of immunized mice, thereby constituting a large percentage of $\mathrm{CD}^{+}$cells. Surprisingly, intravenous immunization did not result in increased numbers of Thy- $1.1^{+}$cells.

The presence of greater numbers of adoptively transferred cells following immunization may have been due to increased survival as well as expansion. The relative contribution of these two effects was not clear. Some expansion of adoptively transferred cells evidently occurred, however, given that the absolute number of Thy- $1.1^{+}$cells determined for the tissues harvested, which accounted for only a small fraction of total mouse tissues, was in excess of the $1 \times 10^{7}$ cells transferred. Although subcutaneous immunization seemed to be more effective than intravenous immunization at expanding adoptively transferred T cells, on the basis of results obtained at day 11 after transfer, it seemed possible that this might reflect different kinetics of expansion produced by the different routes of immunization. To investigate this, we immunized mice once on day 2 after transfer and then harvested tissues at several time points (Fig 2B). We again found that subcutaneous immunization was most effective at expanding $\mathrm{T}$ cells and that their numbers were highest on day 11 after transfer. Intravenous immunization had virtually no effect at any time point, except for a slight increase in the percentage of Thy- $1.1^{+}$cells in the lung on day 6 . We therefore used subcutaneous immunization for subsequent experiments. The specificity of immunization required for expansion was demonstrated by the absence of expansion of anti-H $-2^{\mathrm{d}} \mathrm{T}$ cells in vivo in response to immunization with $\mathrm{H}-2^{\mathrm{k}}$ splenocytes (data not shown).

We next considered whether more effective expansion could be achieved by using allogeneic dendritic cells as the immunogen, because dendritic cells are professional antigen-presenting cells (APCs) that express high levels of MHC class I and II molecules. Mice were subjected to adoptive transfer of alloreactive Thy-1.1 $1^{+}$cells followed by immunization with either allogeneic splenocytes or allogeneic dendritic cells, and tissues were harvested 11 days later. Thy$1.1^{+}$cells constituted about $5 \%$ of spleen, lung, and blood following immunization with splenocytes and about $7-10 \%$ of these tissues following immunization with dendritic cells (Fig. 2C). However, statistical (Kruskal-Wallis test) analysis of this and a repeat experiment indicated that these percentages were not significantly different. Of interest was the high percentage of adoptively transferred cells in popliteal lymph nodes draining the site of footpad immunization, with up to $39 \%$ of lymph node cells being Thy- $1.1^{+}$. Here also there was a trend for immunization with dendritic cells to induce greater numbers of Thy-1.1 $1^{+}$cells, but the difference was not statistically significant. The percentage of Thy $1.1^{+}$cells in lymph node was significantly greater than that in spleen, $P 2<0.01$ (Kruskal-Wallis test).

To further optimize the expansion of Thy $-1.1^{+}$cells in vivo, we investigated the effect of immunizations on different days after transfer and with varying numbers of allogeneic splenocytes. The greatest increase in Thy-1.1 $1^{+}$cells in the spleen was seen following subcutaneous immunization on day 1, 2, or 3 after adoptive transfer (Fig. 3A), and these increases were approximately equivalent. Immunization on day 0 resulted in only slight increases in the percentage of Thy- $1.1^{+}$cells. Although the absolute percentage of adoptively transferred cells was lower than in the previous experiments-reflecting the use of only one immunization instead of three-up to sixfold expansion of Thy-1.1 $1^{+}$ cells occurred with immunization as compared to no immunization.

We next considered whether varying the immunizing dose could affect the expansion of adoptively transferred T cells. Mice were immunized with varying numbers of allogeneic splenocytes on day 2 after transfer of Thy-1.1+ cells, and spleens were harvested on day 11 . Immunization with $5 \times 10^{7}$ allogeneic splenocytes produced the largest expansion of Thy- $1.1^{+}$cells, approximately tenfold greater than in nonimmunized mice (Fig. 3B). A lesser increase in Thy-1.1 $1^{+}$cells (approximately threefold) occurred in mice receiving either $5 \times 10^{6}$ or $2 \times 10^{8}$ allogeneic splenocytes. The lowest immunizing dose, $5 \times 10^{5}$ cells, was ineffective at increasing the percentage of adoptively transferred cells. These results were confirmed in a second experiment. We therefore used an immunizing dose of $5 \times 10^{7}$ splenocytes starting on day 2 in subsequent experiments.

Phenotype and activity of dual-specific T cells. To test the application of this immunization strategy to tumor-reactive lymphocytes, we generated dual-specific $\mathrm{T}$ cells by genetic modification of alloreactive $\mathrm{T}$ cells with a receptor, $\mathrm{MOv}-\gamma$, recognizing the ovarian cancer-associated antigen FBP. Initially, we wished to determine the transgene expression and reactivity of dual-specific cells against both allogeneic cells and FBP. We confirmed that the dual-specific cells expressed the chimeric MOv- $\gamma$ receptor by flow cytometric analysis of the dual-specific T cells following staining with anti-idiotype antibody (Fig. 4A).

The activity and specificity of $\mathrm{T}$ cells generated by genetic modification of T cells from an MLR was determined by a cytokine release assay after overnight coculture with target cells. Alloreactivity of $\mathrm{T}$ cells transduced with either MOv- $\gamma$ or green fluorescent protein (GFP; control) was evident from the high levels of IFN- $\gamma$ secreted in response to the allogeneic target CT26 but not the syngeneic targets MC38 and 24JK (Fig. 4B). FBP reactivity of MOv- $\gamma$-transduced $\mathrm{T}$ cells was demonstrated by their secretion of IFN- $\gamma$ in response to the $\mathrm{FBP}^{+}$cell lines $24 \mathrm{JK}-\mathrm{FBP}$ and IGROV but not the $\mathrm{FBP}^{-}$cell lines $24 \mathrm{JK}$ and 888 . The requirement for MOv- $\gamma$ expression was confirmed by the observation that GFP-transduced T cells did not secrete IFN- $\gamma$ in response to $\mathrm{FBP}^{+}$targets.

Before proceeding with in vivo tumor studies, it was important to first determine whether dual-specific $\mathrm{T}$ cells could expand in vivo in response to allogeneic immunization, as occurred with alloantigenreactive Thy-1.1 $1^{+}$T cells (Fig. $2 \mathrm{~A}$ ). We treated mice with $1 \times 10^{7}$ Thy-1.1 $1^{+}$ dual-specific $T$ cells on day 0 followed by subcutaneous immunization with $5 \times 10^{7}$ allogeneic splenocytes on days 2,5 , and 8 . Spleens were har- 
A

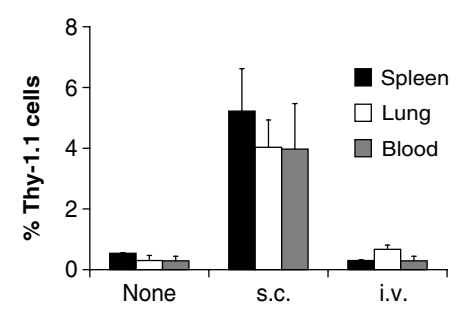

B

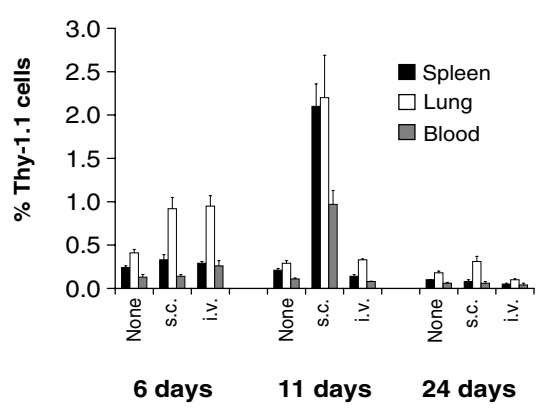

C

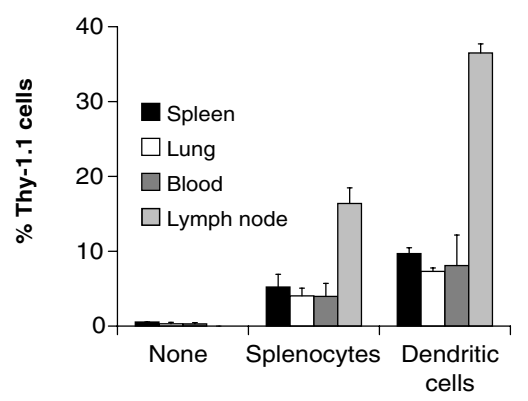

vested on day 11 and analyzed for the percentage of Thy-1.1+ cells. A more than tenfold increase in the percentage of Thy- $1.1^{+}$cells as a result of immunization was consistently observed (Fig. 4C).

Antitumor effect of dual-specific T cells. To determine whether dual-specific $\mathrm{T}$ cells in combination with allogeneic immunization were effective at protecting mice from tumor challenge, we injected mice with dual-specific $\mathrm{T}$ cells, immunized some mice with allogeneic splenocytes on day 2 , and then challenged some mice from each category with tumor on day 9. The majority of mice that received dual-specific $T$ cells and immunization were effectively protected from a challenge dose of $2 \times 10^{4} 24 \mathrm{JK}-\mathrm{FBP}$ tumor cells (Fig. 5A) for the entire observation period of more than 40 days. Mice treated with immunization alone, or injection of dual-specific $\mathrm{T}$ cells alone, were not protected against tumor challenge as compared to untreated mice, demonstrating that both allogeneic immunization and dual-specific T-cell transfer are required for optimal antitumor effect.

To determine whether the combination of dual-specific $T$ cells and immunization was effective at treating tumor cells injected at a later time, mice were injected with $2 \times 10^{5}$ tumor cells, and adoptive transfer of $\mathrm{T}$ cells was done one day later. Immunization with allogeneic splenocytes was then performed on days 3, 6, and 9 after the initial tumor injection. Thus a key element of treatment (immunization) was not applied until tumors were three days old and easily palpable. In three separate experiments, tumor growth in mice receiving dual-specific $\mathrm{T}$ cells and immunization was significantly less in these mice than in untreated mice and mice receiving dual-specific T cells alone (Fig. 5B). Treatment with nonspecific T cells and immunization had no effect, as did treatment of FBP-negative tumor cells with dual-specific cells and immunization. The requirement for both FBP antigen on tumor cells and MOv- $\gamma$ expression on T cells, along with immunization, in order for tumor inhibition to occur was thus clearly demonstrated.
Figure 2. In vivo expansion of adoptively transferred alloreactive T cells. $1 \times 10^{7} \mathrm{H}-2^{\mathrm{d}}$-reactive Thy-1.1 $1^{+} \mathrm{T}$ cells were adoptively transferred into Thy-1.2 $2^{+}$recipients. Some mice received immunization with $5 \times 10^{7} \mathrm{H}-2^{\mathrm{d}}$ splenocytes two, five, and eight days later (A) or only two days later (B). (A) Small numbers of Thy $-1.1^{+}$cells were present in spleen, lung and blood of nonimmunized mice harvested on day 11 after transfer. Mice that received subcutaneous immunization had over tenfold more Thy-1.1+ cells in tissues than nonimmunized mice. In contrast, adoptively transferred cells did not expand in mice that received intravenous immunization. (B) When tissues were harvested at different time points after adoptive transfer and a single immunization two days later, maximal expansion of transferred cells occurred on day 11 , and only with subcutaneous immunization. (C) A comparison of splenocytes with dendritic cells as immunogens revealed these two conditions to be approximately equivalent in their ability to induce expansion of transferred cells in vivo. Thy-1.1+ cells comprised a large percentage of cells in lymph nodes draining the immunization site. Results from one representative experiment of three $(A)$ or two $(B, C)$ are shown, presented as the mean percentage \pm s.e.m. with three mice per group.

Characterization of human dual-specific T cells. To assess the feasibility of this approach for treating human cancers, we attempted to generate human dual-specific T cells by MLR and gene modification with the MOv- $\gamma$ receptor. Functionally, the bulk MOv- $\gamma$-transduced T-cell population was demonstrated to be both alloreactive and FBP reactive (Fig. 6A), whereas nontransduced $\mathrm{T}$ cells were not $\mathrm{FBP}$ reactive. To determine whether individual $\mathrm{T}$ cells within the bulk population were dual specific, we performed cloning by limiting dilution and examined a total of 140 clones in three experiments for reactivity towards allogeneic targets and $\mathrm{FBP}^{+}$targets. Of these clones, $52 \%$ were alloreactive and $68 \%$ of these were FBP reactive. Thus, $35 \%$ of the bulk T-cell population was dual specific. The activity of five representative clones demonstrating dual specificity is evident from the results of a GM-CSF release assay (Fig. 6B). GM-CSF was chosen as the indicator of reactivity because it is secreted by both type 1 and $2 \mathrm{~T}$ cells, in contrast to IFN- $\gamma$, which is secreted predominantly by type $1 \mathrm{~T}$ cells. Notably, the bulk T-cell population was made up largely of $\mathrm{CD} 4^{+} \mathrm{T}$ cells $(83 \%)$, with smaller numbers of $\mathrm{CD}^{+}$cells $(13 \%)$ and some $\mathrm{CD}^{+} \mathrm{CD}^{+}(4 \%)$ and $\mathrm{CD}^{-}{ }^{-} \mathrm{CD} 8^{-}(1 \%)$ cells also present. Of the five representative clones presented here, clones 1,2 , and 3 were $\mathrm{CD} 4^{+}$, clone 4 was $\mathrm{CD} 4^{+} \mathrm{CD} 8^{+}$, and clone 5 was $\mathrm{CD} 8^{+}$.

\section{Discussion}

The potential effectiveness of immunization with TAA as a therapy for cancer is clearly evident from results in mouse tumor models with immunogenic tumors expressing nonself antigen $s^{15}$. However, effective immunization against tumor-associated self antigens is difficult ${ }^{16}$. In humans, too, despite limited success, cancer therapy using TAA has been ineffective ${ }^{4}$. Apparently, most tumor antigens, as currently used, are relatively weak stimulators of immunity. The limited effectiveness of tumor vaccines and other immunotherapeutic strategies for cancer treatment may be partly a consequence of the inability to generate large numbers of activated, high-avidity antitumor T cells.

To address this problem, we have described the generation, by genetic modification of alloreactive cells, of dual-specific $\mathrm{T}$ cells that can expand in vivo in response to a strong immunogenic stimulus (alloantigen) while retaining antitumor reactivity. Alternative specificities for the immunogen component of the dual-specific system are also possible, including viral proteins or even live virus, which would be expected to initiate a vigorous response in vivo. Indeed, a recent report ${ }^{17}$ has described the generation of human T cells that are reactive with GD2 neuroblastoma antigen and Epstein-Barr virus (EBV) in vitro. The authors propose that the prevalence in the human population of EBV in latent form would provide both a ready source of EBV-specific T cells for conversion to dual specificity, and a convenient immunogen that could lead to enhanced survival of adoptively transferred dual-specific T cells. Viral antigens and alloantigens are both potent 
A

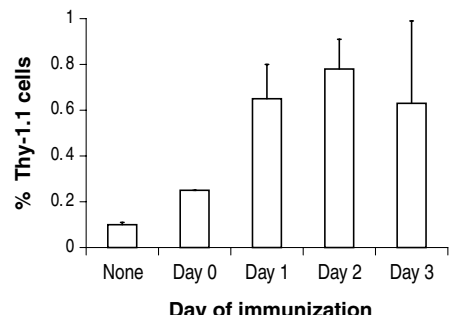

B

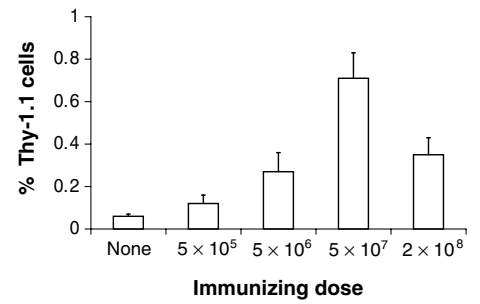

Figure 3. Timing and dose of immunization. (A) Mice received $1 \times 10^{7}$ alloreactive Thy $-1.1^{+} \mathrm{T}$ cells followed by a single subcutaneous immunization with $5 \times 10^{7}$ allogeneic splenocytes on either day $0,1,2$, or 3 after adoptive transfer, or no immunization. The percentage of Thy-1.1 ${ }^{+}$ cells, determined in spleens harvested 11 days after adoptive transfer, was roughly equal in mice immunized on day 1,2 , or 3 and was comparatively lower in mice immunized on day 0 or not immunized. (B) Mice received $1 \times 10^{7}$ alloreactive Thy- $-1.1^{+} \mathrm{T}$ cells followed two days later by subcutaneous immunization with various numbers of allogeneic splenocytes. Some expansion of Thy- $1.1^{+}$cells was seen after $5 \times 10^{6}$ splenocytes, but optimal expansion occurred with a dose of $5 \times 10^{7}$ splenocytes. In both (A) and (B), results are presented as the mean percentage \pm s.e.m. from two experiments with three mice per group in each experiment.

stimulators of immunity, and both that study and ours support the potential of the dual-specific T-cell strategy for the improvement of T-cell immunotherapy. Importantly, the current study supports the feasibility of the approach against tumors in vivo in an animal model.

We examined several parameters important in the expansion of dual-specific cells in vivo and, unexpectedly, found that intravenous immunization did not induce expansion of adoptively transferred cells. The reason for this is not clear, although it is possible that subcutaneously delivered allogeneic cells gained access to the same compartment as adoptively transferred $\mathrm{T}$ cells via the lymphatic system, whereas allogeneic cells delivered intravenously were cleared more rapidly. Intravenous delivery of allogeneic cells has been demonstrated to reduce subsequent responses to alloantigen in other systems ${ }^{18}$.

The most effective immunization procedure among those tested was the subcutaneous injection of $5 \times 10^{7}$ allogeneic splenocytes in the mouse footpads and flank on days 2, 5, and 8 after adoptive transfer. Both alloreactive $\mathrm{T}$ cells in initial experiments and dual-specific $\mathrm{T}$ cells in subsequent experiments expanded in response to immunization to constitute from $3-10 \%$ of total cells in spleen, lung, and blood. Although we did not restrict our calculations to express the numbers of adoptively transferred cells as a percentage of the total $\mathrm{CD} 8^{+}$cells in the tissues, it is likely that in the spleen, for example, Thy- $1.1^{+}$cells make up a large percentage of $\mathrm{CD} 8^{+}$cells.

Our result demonstrating that in vitro-activated and cultured $\mathrm{T}$ cells can expand in vivo following immunization is notable in itself, as in vitro culture may afford the opportunity for manipulation of $\mathrm{T}$ cells to achieve optimal antitumor potential, and yet few studies have used cultured cells in this manner. Interestingly, very high percentages of cells in lymph nodes draining immunization sites consisted of adoptively transferred Thy-1.1+ cells. This would suggest that, although cultured activated $\mathrm{T}$ cells are often thought to have an effector phenotype poorly suited for normal trafficking and function in vivo, there seems to
A

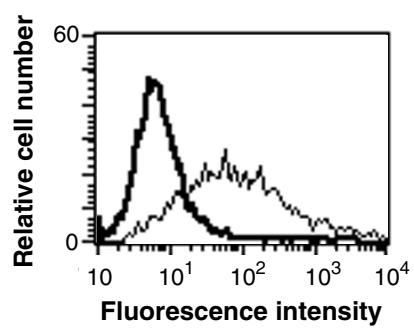

B

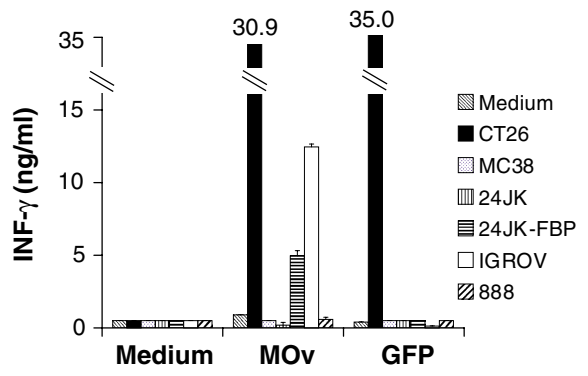

C

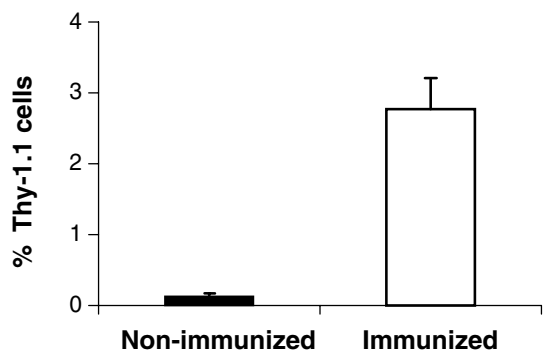

Figure 4. Phenotype, activity, and expansion of dual-specific T cells. (A) Mov- $\gamma$ expression. Dual-specific T cells expressed MOv- $\gamma$, as demonstrated by flow cytometry after staining with anti-idiotype antibody (thin line) compared to isotype control (thick line). Results are representative of many experiments. (B) Dual-specific activity. T cells were cocultured with the target cells listed, and supernatants were assayed by ELISA for the presence of IFN- $\gamma$. Anti-allogeneic activity of both MOv- $\gamma$ - and GFP-transduced T cells was demonstrated by IFN- $\gamma$ secretion in response to the allogeneic target CT26 $\left(\mathrm{H}-2^{\mathrm{d}}\right)$ but not the syngeneic targets MC38 and 24JK $\left(\mathrm{H}-2^{b}\right)$. Anti-FBP reactivity of the MOv- $\gamma$-transduced T cells was evident from their secretion of IFN- $\gamma$ in response to 24JK-FBP but not 24JK cells, and in response to the human $\mathrm{FBP}^{+}$ovarian cancer cell line IGROV but not the FBP- human melanoma cell line 888. T cells did not secrete IFN- $\gamma$ in the absence of target cells. Results of one representative experiment of five are shown. (C) Expansion of dual-specific T cells. Spleens of mice receiving $1 \times 10^{7}$ dual-specific T cells followed by immunization were analyzed for Thy $-1.1^{+}$cells 11 days after transfer. A more than tenfold greater percentage of Thy $-1.1^{+}$cells was consistently observed after immunization as compared to no immunization. Results are mean \pm s.e.m. of three experiments with three mice per group.

be at least a population of cells present that are able to access normal secondary lymphoid tissue and respond to antigen by proliferating.

The tumor specificity here is provided by genetic modification with a chimeric receptor incorporating a single-chain antitumor antibody against ovarian cancer. In previous work from our laboratory, we have described antitumor effects of adoptively transferred mouse $\mathrm{T}$ cells transduced with chimeric receptor ${ }^{14}$. However, the tumors treated in those studies were intraperitoneal xenografts of human ovarian cancer cells or lung metastases of a syngeneic sarcoma expressing FBP. The current study concerns the treatment of subcutaneous tumor, a different disease that is inherently more difficult to treat using adoptively transferred T cells. These considerations-along with our data showing that transduced $\mathrm{T}$ cells alone had little or no effect in the prevention or treatment of subcutaneous tumor, whereas transduced $\mathrm{T}$ cells and 
A

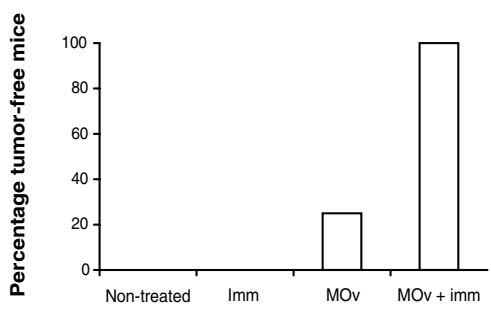

B

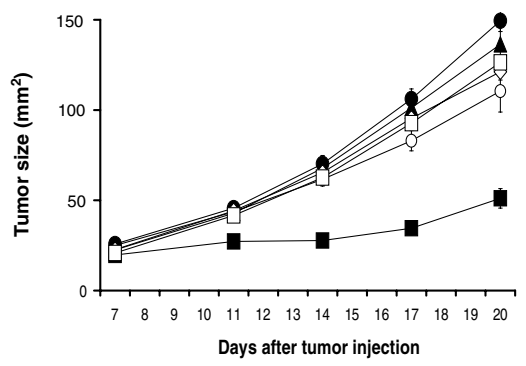

immunization had considerable effect (Fig. 5)—serve to emphasize the extent of the improvement in antitumor efficacy offered by the dualspecific approach. The wider applicability of this approach to other malignancies is evident from work done in our laboratory and others to develop chimeric receptors with specificity for a range of antigens, including those overexpressed on cancers of the colon ${ }^{19,20}$, breast ${ }^{21}$, and kidney $^{22}$ and on tumor vasculature ${ }^{23}$.

Other possible ways to generate dual-specific $\mathrm{T}$ cells include genetic modification of alloreactive cells with $\alpha$ and $\beta$ chains of antitumor $\mathrm{TCR}^{24}$, and modification of tumor-specific $\mathrm{T}$ cells with alloreactive TCR that may then be able to expand in response to immunogen. In this latter approach, knowledge of the antigen responsible for tumor specificity is not necessary. Alternatively, because many $\mathrm{T}$ cells cross-react with alloantigen in addition to their primary TCR specificity ${ }^{25}$, it may be possible to detect antitumor T cells that also respond naturally to allogeneic antigen. This approach might require screening a large panel of allogeneic MHC types to determine the optimal stimulator MHC, but has the advantage of not requiring genetic modification of $\mathrm{T}$ cells.

Because alloantigen produces particularly potent activation and expansion of T cells with other inherent specificities, it is worth considering the possible implications of this approach for the induction of autoimmunity in recipients of therapy. The transfer of large numbers of alloreactive $\mathrm{T}$ cells, and the further generation of alloreactive $\mathrm{T}$ cells in vivo after immunization, could conceivably lead to the activation of $\mathrm{T}$ cells cross-reactive with autoantigens. Of particular concern might be the activation of autoreactive $\mathrm{CD} 4^{+} \mathrm{T}$ cells that could then induce the production of autoreactive antibodies from $\mathrm{B}$ cells. Although there is no direct evidence that this might occur, the possibility is worth bearing in mind.

The dual-specific $\mathrm{T}$ cells used in this study were reactive against two nonself antigens (human FBP and alloantigen), and it is therefore not clear whether this approach could be duplicated for the majority of tumor-associated antigens, which are self antigens. However, studies by other investigators of T-cell ontogeny in double TCR-transgenic mice have demonstrated the ability of the presence of a non-self-reactive TCR to rescue $\mathrm{T}$ cells also bearing a selfreactive TCR from central deletion ${ }^{26}$. Nevertheless, although there is no effective endogenous immune response to FBP, as evidenced by the aggressive growth of FBP-expressing sarcoma at a rate equivalent to the parental FBP-negative cell line, it is still possible that other FBP-induced elements of the immune system, not potent in themselves, contribute to the observed effect.
Figure 5. The combination of adoptive transfer of dual-specific $T$ cells and immunization inhibits tumor growth. (A) Tumor prevention with dual-specific T cells. Mice received either no treatment or MOv- $\gamma$-transduced (dualspecific) T cells $\left(1 \times 10^{7}\right)$; some groups also received subcutaneous immunization with $5 \times 10^{7}$ allogeneic splenocytes two days later. Mice were challenged with tumor seven days after immunization. Tumors grew progressively in untreated mice and in those receiving immunization alone. The majority of mice receiving dual-specific $\mathrm{T}$ cells alone were not protected from tumor challenge, but all mice receiving a combination of dual-specific T cells and immunization were protected from challenge. Dual-specific $T$ cells and immunization was more effective than any other treatment (Kruskal-Wallis test, $P 2<0.05$ ). (B) Tumor treatment with dual-specific T cells. Mice treated with subcutaneous injection of tumor cells, followed by $T$ cells one day later and immunization beginning three days after tumor-cell injection. 24JK-FBP tumors grew progressively in mice receiving either no treatment (filled triangles), GFP-transduced T cells with immunization (filled circles), or dual-specific (MOv) T cells alone (open circles). 24JK-FBP tumor growth was significantly inhibited by the combination of dual-specific $T$ cells and immunization (filled squares). The requirement for FBP expression in tumors for this effect was evident from the absence of 24JK (antigennegative) tumor growth inhibition by dual-specific $T$ cells and immunization (open squares) compared to untreated 24JK tumor (open diamonds). In both $(A)$ and $(B)$, results from one representative experiment of three are shown, presented as the mean \pm s.e.m. with five mice per group.

Also worth considering is that because no effective endogenous immune response is raised against this tumor, 24JK-FBP can be considered a poorly immunogenic tumor in this model. Indeed, the adoptively transferred $\mathrm{T}$ cells detect antigen in an MHC-unrestricted manner, and will not expand in response to tumor antigen in a traditional APCmediated fashion.

It is also difficult to assess the extent of autoimmunity that may be induced in a self-antigen setting where low-level expression of the target TAA may occur on normal tissue. Unfortunately, single-chain antibodies specific for mouse cell-surface TAA are rare, and no chimeric receptors incorporating them are currently available, so it is impossible to answer this question at present. A model system involving a chimeric receptor targeting rat erbB-2 in mice transgenic for rat erbB- 2 is under development in our laboratory and may help provide answers to these questions.

Another question concerns the possible contribution of $\mathrm{T}$ cells reactive to FCS, which was used in the culture of both tumor cells and T cells. Although some FCS-specific T cells could conceivably be generated in culture, we would expect their numbers to be very low in comparison to those of the alloreactive $\mathrm{T}$ cells generated in response to the powerful alloantigen stimulus. Indeed, dual-specific $\mathrm{T}$ cells against MHC class I-positive target cells that have been exposed to FCS, but do not express FBP, show very little activity as measured by IFN $-\gamma$ release (Figs. 1 and 4B). It is unlikely that FCS reactivity contributes substantially to in vivo T-cell expansion, as FCS was not included in our original immunization regimen. The lack of an effect of $\mathrm{T}$ cells against FBPnegative tumors in mice (Fig. 5B) was also an indication that anti-FCS activity does not contribute substantially to antitumor effects. Adding FCS to the immunization schedule did not result in a further increase in the number of adoptively transferred T cells (data not shown). Thus it is not likely that FCS contributes markedly to the observed antitumor effects, and it would not need to be included as an immunogen if this strategy were applied in humans.

We have focused on the effect of immunization on the numbers of dual-specific T cells in vivo, and it is likely that the increase in these cells contributes to the observed antitumor effect. It is also possible, however, that immunization activates dual-specific $\mathrm{T}$ cells that could enhance the antitumor activity of dual-specific T cells. Indeed, activation in this manner might overcome the induction of tolerance to tumor antigen or circumvent active suppression ${ }^{27}$ of tumor-reactive cells.

It is also worth considering that the antitumor effects of dualspecific $\mathrm{T}$ cells may be augmented by help from endogenous recipient cells involved in an immune response to alloantigen. In addition, it is 
A

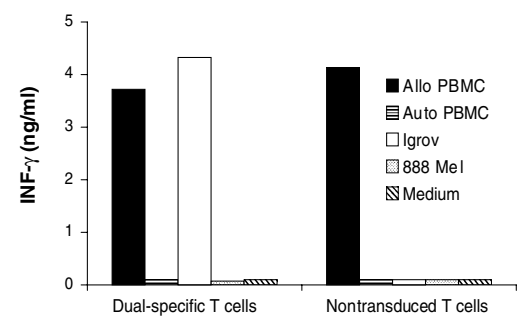

B

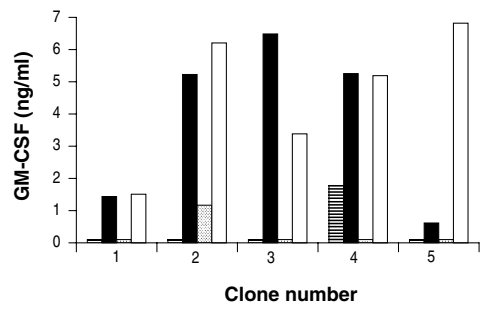

Figure 6. Reactivity of human dual-specific T cells. (A) Human T cells generated against allogeneic antigen and transduced with MOv- $\gamma$ secreted IFN- $\gamma$ in response to both allogeneic PBMC and the $\mathrm{FBP}^{+}$ ovarian cancer cell line IGROV and not in response to autologous PBMC, $\mathrm{FBP}^{-} 888$ melanoma cells, or medium alone, thereby demonstrating the dual specificity of the bulk T-cell population. The absence of IFN- $\gamma$ release by nontransduced T cells incubated with IGROV demonstrates that MOV- $\gamma$ transduction is required for reactivity against FBP. (B) $35 \%$ of transduced T-cell clones from the bulk transduced T-cell population reacted against both allogeneic cells and $\mathrm{FBP}^{+}$target cells. Five representative T-cell clones are shown that secreted GM-CSF in response to both allogeneic PBMC and IGROV, thereby demonstrating that individual T cells can be dual-specific. Smaller amounts of GM-CSF were secreted in response to autologous PBMC and the 888 melanoma cell line. Nontransduced clones did not secrete GM-CSF in response to $\mathrm{FBP}^{+}$cells (data not shown).

Generation, characterization, and cloning of human dual-specific T cells was done three times with similar results.

possible that adoptively transferred mono-specific T cells (alloreactive only) present in the transferred cells contribute indirectly to enhancing the antitumor activity of chimeric receptor-expressing $\mathrm{T}$ cells following immunization. The relative contribution from these indirect effects is not clear from this study, although there is probably a significant mechanistic contribution resulting from the large increase in the numbers of dual-specific cells following immunization.

The adoptively transferred bulk mouse T-cell population is probably composed of a mixture of cells specific for alloantigen alone or FBP alone in addition to dual-specific T cells. The relative increase in these separate populations in vivo following immunization is not known. It is likely, however, that dual-specific T cells are present in vivo for at least 11 days, because T cells re-isolated from spleens and lymph nodes of recipient mice at this time point were able to proliferate in response to re-stimulation with allogeneic splenocytes in vitro in the presence of the neomycin analog G418, which selected for transduced $\mathrm{T}$ cells, and to retain chimeric MOv- $\gamma$ expression and dual reactivity (data not shown).

It is not clear from this study what contribution different T-cell subsets make to the observed effect in mice, although it is likely that CD8 ${ }^{+}$ $\mathrm{T}$ cells contribute substantially because these represent a large percentage $(>95 \%)$ of the mouse $\mathrm{T}$ cells used for adoptive transfer. Investigators have consistently found a preponderance of $\mathrm{CD} 8^{+} \mathrm{T}$ cells following stimulation of mouse splenocytes by MLR or lectin, although the reason for this polarization is not clear. In humans, by contrast, MLR results in mainly (often $>80 \%$ ) $\mathrm{CD}^{+}$cells in some cases. Although $\mathrm{CD}^{+}$may contribute to antitumor reactivity, in applying this approach clinically it might be advantageous to include strategies that involve enrichment of $\mathrm{CD} 8^{+}$cells or selection of an immunogen that preferentially expands $\mathrm{CD}^{+}$cells.

The strategy described here requires culturing of T cells to enrich for alloreactive cells. Activation in culture is also necessary to achieve integration of retroviral vectors in their current form. This can potentially decrease the function of $\mathrm{T}$ cells in vivo following adoptive transfer as compared to noncultured T cells. Earlier investigators have observed other examples of decreased function, whereby transfer of cultured allogeneic $\mathrm{T}$ cells reduced their capacity to induce graft-versus-host disease in allogeneic recipient animals ${ }^{28,29}$. In our approach, large numbers of adoptively transferred $\mathrm{T}$ cells persist (and probably expand), and in vitro reactivity is high; however, improvements could perhaps be achieved by modifying the culture or transduction conditions or by minimizing in vitro culture. To this end, an amended strategy involving isolation of naive $\mathrm{T}$ cells using appropriate tetramers and minimal culture with retroviral or lentiviral vectors might be fruitful.

In conclusion, many hurdles must be overcome in order to achieve effective immunization against tumor antigens, not the least of which is the need to effectively circumvent self-tolerance to TAAs that are generally expressed in other, normal tissues. By delegating the responsibility for expansion and activation to a highly responsive yet separate receptor from that encoding tumor reactivity, it may be possible to overcome the problem of weak tumor immunogenicity. The production of dualspecific $\mathrm{T}$ cells provides an alternative strategy for vaccine development and immunization because it offers a means to generate a potent reaction against poorly immunogenic antigens.

\section{Experimental protocol}

Cell lines and mice. 24JK is a methylcholanthrene-induced sarcoma of C57BL/6 mice, and 24JK-FBP was derived from this line by transduction with cDNA encoding human FBP. B16 is a melanoma and MC38 is a colon adenocarcinoma of C57BL/6. CT26 is a colon carcinoma and BALB/c $3 T 3$ is a fibroblast line of $\mathrm{BALB} / \mathrm{c}$ mice. IGROV is a $\mathrm{FBP}^{+}$human ovarian cancer cell line, and 888 is a FBPhuman melanoma cell line. The retroviral producer cell lines used were GP+E86 for mouse studies and PG13 for experiments with human cells. The retroviral construct encoding the chimeric receptor, $\mathrm{MOv}-\gamma$, was composed of extracellular single-chain MOv18 anti-ovarian carcinoma antibody specific for human $\mathrm{FBP}^{30,31}$ linked to the transmembrane and cytoplasmic domains of the human Fc receptor $\gamma$ chain. This chimeric receptor was cloned into the vector pSAMEN $^{32}$, where expression was driven by the long terminal repeat promoter region of the Moloney murine leukemia virus. A neomycin-resistance gene (neomycin phosphotransferase) is included in this vector under the transcriptional control of an internal ribosomal entry site. Previous studies characterizing the parental MOv18 antibody ${ }^{31}$ indicate that it has an affinity constant of $2 \times 10^{8} \mathrm{M}^{-1}$ and that the number of antigenic determinants on human ovarian cancer cells is on the order of $1 \times 10^{7}$.

Producer cell lines were generated by genetic modification with the pSAMEN vector encoding either the chimeric receptor MOv- $\gamma$ or GFP, using a method similar to that previously reported ${ }^{20}$, followed by transduction of GP+E86 and PG13. All cell lines were cultured in RPMI 1640 medium supplemented with $10 \%$ heat-inactivated FCS, $2 \mathrm{mM}$ glutamine, $100 \mathrm{U} / \mathrm{ml}$ penicillin, $100 \mu \mathrm{g} / \mathrm{ml}$ streptomycin, $1.25 \mu \mathrm{g} / \mathrm{ml}$ amphotericin $\mathrm{B}, 5 \mu \mathrm{g} / \mathrm{ml}$ gentamycin, and $5 \times 10^{-5} \mathrm{M}$ 2-mercaptoethanol. Mice used in these experiments were $\mathrm{C} 57 \mathrm{BL} / 6$ and BALB/c produced at the Animal Production Area, National Cancer Institute (Frederick, MD). Thy-1.1 C57BL/6 mice (B6.PL-Thy ${ }^{\mathrm{a}} / \mathrm{Cy}$ ) were obtained from Jackson Laboratories (Bar Harbor, ME). The experimental design was approved by the Animal Experimental Ethics Committee, National Insitutes of Health.

Antibodies and flow cytometry. Antibodies $(1 \mu \mathrm{g})$ specific for CD4, CD8, $\mathrm{H}-$ $2 \mathrm{~K}^{\mathrm{b}}$, AND Thy-1.1, and rat IgG isotype controls (Pharmingen, San Diego, CA), were incubated with $1 \times 10^{6}$ cells. The cells were then washed in PBS and assayed by flow cytometry. For the detection of chimeric $\mathrm{MOv}-\gamma$ receptor, cells were incubated with a phycoerythrin-conjugated antibody directed at the idiotype of the single-chain antibody making up the extracellular portion of the receptor.

Generation of alloreactive and dual-specific $\mathrm{T}$ cells. Mouse alloreactive $\mathrm{T}$ cells were generated by MLR involving coculture of $2 \times 10^{6} \mathrm{C} 57 \mathrm{BL} / 6$ Thy- $1.1^{+}$splenocytes with $2 \times 10^{6}$ irradiated (2,000 Rad) BALB/c splenocytes per well in 24-well plates. Human interleukin-2 (IL-2; $60 \mathrm{IU} / \mathrm{ml}$ ) (Chiron Corp, Emeryville, CA) was added on day 2 and every second day thereafter. Cultures were maintained 
at a cell density of $0.5 \times 10^{6}-3 \times 10^{6}$ cells per well. Cells were restimulated every 7 days by seeding at $5 \times 10^{5} \mathrm{~T}$ cells per well and adding $2 \times 10^{6}$ irradiated $\mathrm{BALB} / \mathrm{c}$ splenocytes and IL-2. T cells were used for adoptive transfer on day 5 or 6 after one or two re-stimulations. Dual-specific mouse T cells were generated by MLR in similar conditions, except that $1 \times 10^{5}$ non-irradiated GP+E86 retroviral packaging cells were preseeded in $1 \mathrm{ml}$ into each well the night before the MLR. Subsequent re-stimulations of transduced mouse $\mathrm{T}$ cells were done in the presence of the neomycin analog G418 (500 $\mu \mathrm{g} / \mathrm{ml})$. Dual-specific human T cells were generated in MLR similarly to mouse dual-specific cells, except that irradiated allogeneic PBMC were used as stimulators and retroviral supernatant from PG13 producer lines was used as described ${ }^{20}$ for two transductions.

Determining percentage of Thy-1.1 $1^{+}$cells in tissues. Spleens and popliteal lymph nodes were harvested and crushed; lung and tumor were digested with hyaluronidase $(100 \mu \mathrm{g} / \mathrm{ml})$, collagenase $(1 \mathrm{mg} / \mathrm{ml})$, and DNase $(30 \mathrm{U} / \mathrm{ml})$ (all from Worthington Biochemical, Lakewood, NJ) for six hours. $5 \times 10^{6}$ cells were stained with phycoerythrin-conjugated anti-Thy-1.1 and analyzed by flow cytometry. The amount of Thy-1.1 $1^{+}$cells present in each tissue was determined as the percentage of total cells in that tissue.

Adoptive transfer of $\mathrm{T}$ cells and immunization. For tumor-prevention studies, mouse $\mathrm{T}$ cells were harvested from 24-well plates, washed twice in PBS, and resuspended at $2.5 \times 10^{7}$ cells $/ \mathrm{ml}$, and $0.4 \mathrm{ml}$ were injected intravenously via the tail vein into mice. For tumor treatment studies, $1 \times 10^{6} \mathrm{~T}$ cells $\left(\right.$ or $3 \times 10^{6} \mathrm{~T}$ cells in some experiments) in $0.4 \mathrm{ml}$ of PBS were injected intravenously. Unless otherwise stated, immunization of mice was done using $5 \times 10^{7}$ allogeneic splenocytes in $0.2 \mathrm{ml} \mathrm{PBS}$, either intravenously (tail vein) or subcutaneously ( $0.05 \mathrm{ml}$ foot- pads and $0.1 \mathrm{ml}$ flank) on days 2,5 , and 8 after adoptive transfer. In some experiments, $1 \times 10^{7}$ allogeneic dendritic cells were used as immunogen. Dendritic cells were generated from bone marrow isolated from the legs of BALB/c mice and cultured in murine IL- 4 and GM-CSF, as reported previously $y^{33}$.

Cytokine release assays. T-cell reactivity against alloantigen and FBP was determined by co-incubating $1 \times 10^{5} \mathrm{~T}$ cells with $1 \times 10^{5}$ target cells in 96-well plates overnight and then assaying the supernatants for IFN- $\gamma$ or GM-CSF by enzymelinked immunosorbent assay (ELISA) using commercially available reagents according to the manufacturers instructions (Endogen, Woburn, MA).

Tumor studies. For tumor-prevention studies, mice were injected with $1 \times 10^{7} \mathrm{~T}$ cells on day 0 and immunized by subcutaneous injection with $5 \times 10^{7}$ non-irradiated allogeneic splenocytes on day 2 . Mice were challenged subcutaneously with either $24 \mathrm{JK}$ or $24 \mathrm{JK}-\mathrm{FBP}$ cells on day 9 , and tumor growth was monitored. For tumor-treatment studies, mice were irradiated with a sublethal dose of radiation $(600 \mathrm{cGy})$ to delay the onset of an endogenous antibody response to human FBP that would interfere with the interaction between FBP on tumor and anti-FBP receptor on dualspecific $\mathrm{T}$ cells. On the same day, mice were injected subcutaneously with $2 \times 10^{5}$ tumor cells. Mice received adoptive transfer of $\mathrm{T}$ cells one day later. Immunization with $5 \times 10^{7}$ irradiated (2000 cGy) allogeneic splenocytes was performed on days 3,6 , and 9 after tumor injection.

Competing interests statement

The authors declare that they have no competing financial interests.

Received 10 April 2002; accepted 19 September 2002
1. Henderson, R.A. \& Finn, O.J. Human tumor antigens are ready to fly. Adv. Immunol. 62, 217-256 (1996)

2. Boon, T., Coulie, P.G. \& Van den, E.B. Tumor antigens recognized by T cells. Immunol. Today 18, 267-268 (1997)

3. Wang, R.F. \& Rosenberg, S.A. Human tumor antigens for cancer vaccine development. Immunol. Rev. 170, 85-100 (1999).

4. Rosenberg, S.A. et al. Immunologic and therapeutic evaluation of a synthetic peptide vaccine for the treatment of patients with metastatic melanoma. Nat. Med. 4, 321-327 (1998).

5. Greenberg, P.D. \& Riddell, S.R. Deficient cellular immunity-finding and fixing the defects. Science 285, 546-551 (1999).

6. Rooney, C.M. et al. Infusion of cytotoxic T cells for the prevention and treatment of Epstein-Barr virus-induced lymphoma in allogeneic transplant recipients. Blood $\mathbf{9 2}$, 1549-1555 (1998).

7. Rosenberg, S.A. et al. Use of tumor-infiltrating lymphocytes and interleukin-2 in the immunotherapy of patients with metastatic melanoma. A preliminary report. $\mathrm{N}$. Engl. J. Med. 319, 1676-1680 (1988).

8. Li, Q. \& Chang, A.E. Adoptive T-cell immunotherapy of cancer. Cytokines Cell. Mol. Ther. 5, 105-117 (1999)

9. Spiess, P.J., Yang, J.C. \& Rosenberg, S.A. In vivo antitumor activity of tumor-infiltrating lymphocytes expanded in recombinant interleukin-2. J. Natl. Cancer Inst. 79, 1067-1075 (1987)

10. Hanson, H.L. et al. Eradication of established tumors by $\mathrm{CD}^{+} \mathrm{T}$ cell adoptive immunotherapy. Immunity 13, 265-276 (2000).

11. Rosenberg, S.A. et al. Gene transfer into humans: immunotherapy of patients with advanced melanoma using tumor-infiltrating lymphocytes modified by retroviral gene transduction. N. Engl. J. Med. 323, 570-578 (1990).

12. Sherman, L.A. \& Chattopadhyay, S. The molecular basis of allorecognition. Annu. Rev. Immunol. 11, 385-402 (1993)

13. Suchin, E.J. et al. Quantifying the frequency of alloreactive T cells in vivo: new answers to an old question. J. Immunol. 166, 973-981 (2001).

14. Hwu, P. et al. Lysis of ovarian cancer cells by human lymphocytes redirected with a chimeric gene composed of an antibody variable region and the $\mathrm{Fc}$ receptor $\gamma$ chain. J. Exp. Med. 178, 361-366 (1993).

15. Specht, J.M. et al. Dendritic cells retrovirally transduced with a model antigen gene are therapeutically effective against established pulmonary metastases. J. Exp. Med. 186, 1213-1221 (1997)

16. Sherman, L.A. et al. Strategies for tumor elimination by cytotoxic T lymphocytes. Crit. Rev. Immunol. 18, 47-54 (1998).

17. Rossig, C., Bollard, C.M., Nuchtern, J.G., Rooney, C.M. \& Brenner, M.K. Epstein-Barr virus-specific human $T$ lymphocytes expressing antitumor chimeric T-cell receptors: potential for improved immunotherapy. Blood 99, 2009-2016 (2002).

18. Heeg, K. \& Wagner, H. Induction of peripheral tolerance to class I major histocompatibility complex (MHC) alloantigens in adult mice: transfused class I MHC- incompati- ble splenocytes veto clonal responses of antigen-reactive Lyt-2+ $\mathrm{T}$ cells. J. Exp. Med. 172, 719-728 (1990)

19. Darcy, P.K. et al. Redirected perforin-dependent lysis of colon carcinoma by ex vivo genetically engineered CTL. J. Immunol. 164, 3705-3712 (2000).

20. Daly, T. et al. Recognition of human colon cancer by $T$ cells transduced with a chimeric receptor gene. Cancer Gene Ther. 7, 284-291 (2000).

21. Altenschmidt, U., Klundt, E. \& Groner, B. Adoptive transfer of in vitro-targeted, activated T lymphocytes results in total tumor regression. J. Immunol. 159, 5509-5515 (1997).

22. Weijtens, M.E., Willemsen, R.A., Valerio, D., Stam, K. \& Bolhuis, R.L. Single chain $\mathrm{lg} / \gamma$ gene-redirected human $T$ lymphocytes produce cytokines, specifically lyse tumor cells, and recycle lytic capacity. J. Immunol. 157, 836-843 (1996).

23. Kershaw, M.H. et al. Generation of gene-modified T cells reactive against the angiogenic kinase insert domain-containing receptor (KDR) found on tumor vasculature. Hum. Gene Ther. 11, 2445-2452 (2000).

24. Clay, T.M., Custer, M.C., Sachs, J., Hwu, P. Rosenberg, S.A. \& Nishimura, M.I. Efficient transfer of a tumor antigen-reactive TCR to human peripheral blood lymphocytes confers anti-tumor reactivity. J. Immunol. 163, 507-513 (1999).

25. Matis, L.A., Sorger, S.B., McElligott, D.L., Fink, P.J. \& Hedrick, S.M. The molecular basis of alloreactivity in antigen-specific, major histocompatibility complex-restricted T cell clones. Cell 51, 59-69 (1987).

26. Legrand, N. \& Freitas, A.A. CD8 ${ }^{+}$T lymphocytes in double $\alpha \beta$ TCR transgenic mice. I. TCR expression and thymus selection in the absence or in the presence of self-antigen. J. Immunol. 167, 6150-6157 (2001).

27. Shevach, E.M. Suppressor T cells: rebirth, function and homeostasis. Curr. Biol. 10, R572-R575 (2000)

28. Drobyski, W.R., Majewski, D., Ozker, K. \& Hanson, G. Ex vivo anti-CD3 antibody-activated donor $T$ cells have a reduced ability to cause lethal murine graft-versus-host disease but retain their ability to facilitate alloengraftment. J. Immunol. 161, 2610-2619 (1998)

29. Weijtens, M.U., van Spronsen, A., Hagenbeek, A.U., Braakman, E. \& Martens, A. Reduced graft-versus-host disease-inducing capacity of T cells after activation, culturing, and magnetic cell sorting selection in an allogeneic bone marrow transplantation model in rats. Hum. Gene Ther. 13, 187-198 (2002).

30. Coney, L.R. et al. Cloning of a tumor-associated antigen: MOv18 and MOv19 antibodies recognize a folate-binding protein. Cancer Res. 51, 6125-6132 (1991).

31. Miotti, S.U. et al. Characterization of human ovarian carcinoma-associated antigens defined by novel monoclonal antibodies with tumor-restricted specificity. Int. J. Cancer 39, 297-303 (1987).

32. Chuah, M.K., Vandendriessche, T. \& Morgan, R.A. Development and analysis of retroviral vectors expressing human factor VIII as a potential gene therapy for hemophilia A. Hum. Gene Ther. 6, 1363-1377 (1995).

33. Kershaw, M.H. et al. Immunization against endogenous retroviral tumor-associated antigens. Cancer Res. 61, 7920-7924 (2001). 